\title{
System-Level Design Considerations for Carbon Nanotube Electromechanical Resonators
}

\author{
Christian Kauth, Marc Pastre, Jean-Michel Sallese, and Maher Kayal
}

Electronics Laboratory, Ecole Polytechnique Fédérale de Lausanne, 1015 Lausanne, Switzerland

Correspondence should be addressed to Christian Kauth; christian.kauth@epfl.ch

Received 24 May 2013; Accepted 19 September 2013

Academic Editor: Andrea Cusano

Copyright (C) 2013 Christian Kauth et al. This is an open access article distributed under the Creative Commons Attribution License, which permits unrestricted use, distribution, and reproduction in any medium, provided the original work is properly cited.

Despite an evermore complete plethora of complex domain-specific semiempirical models, no succinct recipe for large-scale carbon nanotube electromechanical systems design has been formulated. To combine the benefits of these highly sensitive miniaturized mechanical sensors with the vast functionalities available in electronics, we identify a reduced key parameter set of carbon nanotube properties, nanoelectromechanical system design, and operation that steers the sensor's performance towards system applications, based on open- and closed-loop topologies. Suspended single-walled carbon nanotubes are reviewed in terms of their electromechanical properties with the objective of evaluating orders of magnitude of the electrical actuation and detection mechanisms. Open-loop time-averaging and $1 \omega$ or $2 \omega$ mixing methods are completed by a new $4 \omega$ actuation and detection technique. A discussion on their extension to closed-loop topologies and system applications concludes the analysis, covering signal-to-noise ratio, and the capability to spectrally isolate the motional information from parasitical feedthrough by contemporary electronic read-out techniques.

\section{Introduction}

Since their discovery [1] and tremendous boost in popularity two decades ago [2], carbon nanotubes (CNTs) incited researchers from various domains to investigate, among others, their electrical and mechanical properties. Their high integrity, quality factor, and small dimensions are white hope for the single-walled carbon nanotubes' (SWNTs) advance to applications, such as electromechanical resonators for RF transmission and reception, voltage-controlled oscillators, or single molecule weighing [3]. First models emerged and kept refining up to reach an impressive complexity, sometimes beyond the scope of circuit design, that generally prefers to trade model complexity for simplicity and clarity. To date, the library of models that describe in detail partial CNT behaviour under specific conditions has reached a critical volume. Focusing on strictly relevant parameters is not a trivial task for nanoelectromechanical system (NEMS) designers anymore. This is an unfortunate fact, when one considers that the relatively low device yield and rare occasions to observe the desired phenomena, already protract the CNT-NEMS advancement to system-level industrial applications.

In this scope, our work reviews the state-of-the-art CNTNEMS devices from a system-level point of view and draws clear guidelines on CNT parameter selection and device biasing to foster the CNT's operation as a mechanical component within electronic circuits that implement versatile functionalities. Never measured orders of magnitude of the signals encoding motional and parasitical information are extracted for those deliberate designs. This analysis leads to a systematic completion of the recent open-loop readout techniques based on time-averaging [4] and $1 \omega$ [5] or $2 \omega$ [6] mixing, by a new $4 \omega$ mixing technique. To pave the way to appealing sensor applications, closed-loop oscillators, locking on the NEMS' motional signal is introduced and discussed with respect to the capability of circuit-level frontends, to amplify and filter these signals out of the noisy background for further processing. Crucial effects, steering the overall system behaviour, are identified; others are shown to be negligible. The approach remains at high level with major emphasis on principles and 
orders of magnitude, while the underlying physics is briefly highlighted, referring to the related state-of-the-art literature.

Section 2 formalizes the tunable suspended clampedclamped SWNT [7] with its electrodes as an electromechanical multiport, core component of any system application. A possible operation of this multiport within a closed-loop oscillator structure is outlined. The functionality of electrical (Section 3) and mechanical (Section 4) ports is first examined isolatedly in their respective energy domains, then in conjunction (Section 5), opening the gate to electronic actuation and sensing of mechanical phenomena. The strength and frequency of the dominant effects are assessed and may serve as reference work to future NEMS application designers. The capability of low-noise electronic circuits to sense and amplify the NEMS' motional information, in terms of spectral signal separation from parasitical feed-through and minimum detectable signal, is assessed for open-loop resonators (Section 6) and all-purpose closed-loop topologies (Section 7), leading to promising conclusions (Section 8) for emerging NEMS sensors.

\section{The NEMS as an Electromechanical Multiport}

Motivated by remarkable electromechanical sensing properties, the system of interest operates the SWNT as channel material, contacted in a transistor configuration (Figure 1). Mechanical degrees of freedom occur when the channel is suspended and allow for frequency tuning via straining [8]. The CNTs' current source behaviour (Figure 2) lends itself to current-mode readout. Maximal motional signal strength (piezoresistive and motional field effect), minimal parasitic signal strength (gate-drain capacitance, noise), and minimum intrinsic signal loss (output impedance) are desirable from a system-level point of view and become NEMS device design objectives.

With force and voltage (potential difference) as acrossvariables, velocity and current as through-variables, in the mechanical and electrical energy domains respectively, this electromechanical system presents two electrical (gate and source potential) and two mechanical (source and channel positions) degrees of freedom to steer interdomain energy transfer, if the drain is chosen to be the electrical (potential) and mechanical (position) reference. This decision roots in the fact that the movable, frequency tuning, electrode (source) presents an elevated parasitic capacitance, which hampers high frequency readout, necessary for closedloop applications as depicted in Figure 1. The CNT is actuated via an electrostatic force pulling from the gate electrode. Its motion modulates the current flowing through it by nanoamperes. This current is sensed at the drain and amplified by a low-noise frontend. Given the CNTs' ultra-high resonance frequencies, noise is integrated over a considerable bandwidth and a bandpass filter is required to put things right (Section 7). This filter also attenuates undesired parasitical feed-through signals from the actuating electrode (Section 6). Gain and phase regulation close the loop by ensuring proper oscillation buildup and stabilization. Molecules binding to the tube or strain induced via the source

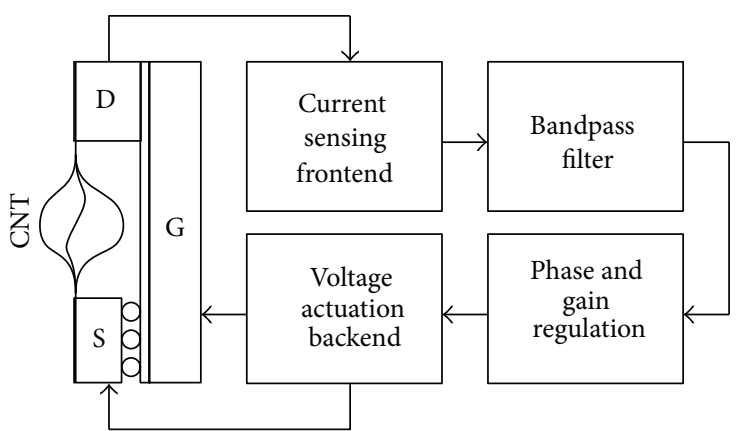

FIGURE 1: Sensing resonator NEMS in a generic closed-loop oscillator topology.

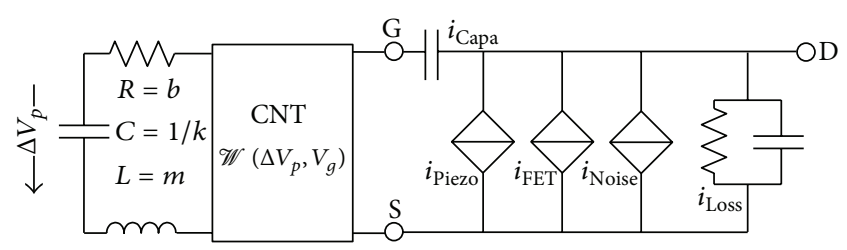

FIGURE 2: NEMS as electromechanical two- motional contributions (piezoresistive and motional-FET) to the drain current.

may alter the CNT's resonance frequency, entailing sensor and voltage controlled oscillator (VCO) applications [3].

With the mechanical properties of SWNTs being primarily defined by the strong covalent in-plane $s p^{2}$ bonds ( $\sigma$-bonds), while the electronic properties depend almost exclusively on the delocalized $\pi$-states, the system qualifies for a preliminary isolated study in the purely electrical and mechanical domains. The combination of electromechanical effects will be studied subsequently and reveals the efficient operation of SWNT NEMS as electromechanical transducers and sensors.

\section{Carbon Nanotube Field Effect Transistor}

This section highlights the most pertinent system-level parameters of carbon nanotube field effect transistors (CNFET), the CNT's projection into the purely electrical domain. Since their first demonstration at room temperature [9], a heap of related work has been reported on SWNT electronics [10], ballistic effects [11], their advance to $\mathrm{GHz}$ frequencies [12], and circuit models [13]. To countersteer system designer's resentment felt against the plethora of state-of-theart findings, which reveal that apparently similar devices may behave very differently, orders of magnitude of the different CNFET phenomena are extracted and intend to guide system designers through proper device selection, DC bias, and AC operation, summarized at the end of this section.

\subsection{CNT Properties}

3.1.1. Electronic Nature (Affected by Chirality). The metallic or semiconducting nature of SWNTs is defined by their chirality. The lack of chirality control [14] in the growth processes currently constrains CNT-based circuits to small sizes for yield 
considerations. Without any selection [15], only two tubes out of three can be expected to possess the desirable conductance controllability property. The electronic properties of SWNTs originate from the band structure of graphene, confined to a 2D rolled-up stripe. Under the nearest neighbour tight binding approximation [16], the valence and conduction $\pi$ bands of graphene intersect in 6 singular K-points within the first Brillouin zone, awarding graphene the semimetal designation. Via zone folding, the Brillouin zone for SWNTs is quantized and its dispersion relation presents a bandgap if none of the K-points belongs to the quantized zone, which is statistically the case for two SWNTs out of three. A refined model [17] predicts for small diameter tubes the opening of a narrow bandgap in otherwise metallic tubes, but thermal noise restricts their exploitation to cryogenic temperatures.

3.1.2. Bandgap (Affected by Diameter). While the electrical nature is defined by the chirality, the bandgap details of semiconducting tubes are mainly imposed by the diameter $d$. Tremendous progress has been made on diameter control [18] and allows for synthesis of large $(d>2 \mathrm{~nm})$ or narrow $(d<$ $1 \mathrm{~nm}$ ) diameter tube distributions with standard deviations of less than $0.1 \mathrm{~nm}$, translating into precisely controlled energy gaps in the $0.5-0.8 \mathrm{eV}$ range [18]. The SWNT diameter will enter the design parameter set and fixes the bandgap approximately via [19]

$$
E_{g}[\mathrm{eV}]=\frac{0.8}{d[\mathrm{~nm}]},
$$

implying larger bandgaps for narrow tubes. As a consequence, large diameter tubes are generally favoured for their lower contact resistance and higher current drive capabilities, while narrow diameter tubes are beneficial for low-power targets.

\subsubsection{The Ultimate Resistance in CNT and Saturation Current} (Affected by Length). The intrinsic resistance of metallic SWNTs is composed of a quantum resistance described by the Landauer-Büttiker formula [20], completed by a Drude-like resistance. The quantum resistance originates from the severe reduction of the large number of modes in the macroscopic contacts to solely two quasidegenerate bands with van Hove singularities, each of which can carry spin up or down, in the 1D SWNT. This limits the maximum conductance of SWNTs to $G_{0}=4 e^{2} / h \approx 155 \mu \mathrm{S}$. Under the hypothesis of perfect ohmic contacts, which will be discussed in Section 3.2.1, semiconducting SWNTs present close to zero conductance if the Fermi level falls into the bandgap and can reach $G_{0}$ under ballistic transport. With mean free paths up to $\mu \mathrm{m}$ scale [10], SWNTs are ballistic [11] under low bias and length scales below hundreds of $\mathrm{nm}$. Shorter devices will switch faster due to time-of-flight considerations but will never exceed this upper conductance bound. In longer channels, localized lattice defects [21] and long range potential fluctuations in the oxide may cause elastic scattering. Combined to inelastic low-bias acoustical electron-phonon scattering, these reflections translate into a series Drude-like resistance. Consequently, the voltage starts to drop along the SWNT channel and parameters such as charge mobility and resistivity can be defined with best of breed values of $10^{4} \cdots 10^{5} \mathrm{~cm}^{2} / \mathrm{Vs}[22,23]$ and $10^{-6} \Omega \mathrm{cm}$ [10]. As the channel resistance grows larger than the contact resistance, very long devices might behave more like bulk-switching MOSFETs, where transport is dominated by drift of carriers inside a charge gradient. The contact resistance to CNTs shows to be rather high in practice, often above $M \Omega$. This can be mediated by proper choice of the electrode material to have conduction states which extend through both the CNT and the metal. Further, defects can be intentionally introduced either on the CNT or the metal, to cause scattering at the interface [24], which results in a reduced contact resistance of about $100 \mathrm{k} \Omega$ [25]. This sum of the linear regime on-resistance and the contact resistance can be directly measured under low drain-source bias. While conductances close to the upper bound can be achieved by freezing out the Drude resistance, room-temperature values of $10 \%$ and $50 \%$ of this upper limit have been demonstrated for long $(L=3 \mu \mathrm{m})$ and short $(L=300 \mathrm{~nm})$ devices [11], respectively. Under high bias, optical electron-phonon excitation sets in, resulting in a linear increase of the Drude resistance [26] and implying a saturation asymptote. The maximum current through SWNTs was indeed shown to be $\sim 25 \mu \mathrm{A}[26,27]$. This asymptote was circumvented in extremely short devices $(L \sim 10 \mathrm{~nm})$ with current values of $60 \mu \mathrm{A}$ and no sign of saturation [28]. Saturation currents of $\mu \mathrm{A}$ are considered an indicator of acceptable device selection, design, and operation for current mode sensor applications. It is noteworthy to mention that this current saturation boosts the CNT's output impedance and transforms the CNT's linear regime resistive behaviour (under small $V_{d s}$ ) into an active, controllable current source behaviour (under larger $V_{d s}$ ) [10].

3.2. CNFET Design. Although careful choice of the relevant SWNT properties is essential, it is not sufficient for proper device functionality.

3.2.1. Contact Type (Affected by Contact Material). The Schottky or ohmic nature of the contacts is greatly determined by the contact material. Due to the unique $1 \mathrm{D}$ structure and a quasi 0D interface of SWNTs, the interface states are not strong enough to pin the Fermi level [29]. The height of the Schottky barrier at the metal-CNT interface therefore depends strongly on the metal work function. Desired ohmic contacts for high device performance can be achieved for, among others, Al, Cr, or Pd contacts [11]. Smaller bandgaps favour the formation of ohmic contacts for at least one type of carrier. In accordance with (1), a clear diameter and Schottkybarrier height dependence of the apparent on-state resistance were demonstrated [30]. SWNT diameters well below $1 \mathrm{~nm}$ have bandgaps that approach $1 \mathrm{eV}$, making the formation of Schottky barriers at the interface more likely and boosting the apparent SWNT resistance to $\mathrm{M} \Omega$ values [31].

3.2.2. Controllability (Affected by Oxide Thickness and GateCNT Distance). The device's controllability via the gate electrode strongly depends on the effective electrode overlap with the channel, the dielectric constant of the insulator, and the gate's distance to the possibly suspended channel. While 
cylindrical all-around thin-oxide [32] and electrolytic [33] gates provide best controllability [34], electrostatic actuation for motion claims for an asymmetrical gate structure. Thin high-k oxides, acting on a wide section of the device, enhance controllability [35], but such nm thick oxides might leak pA [36]. The exact mechanisms of channel control are discussed subsequently.

3.3. Device Bias. Once the design is accomplished, there remain two electrical parameters to regulate the device characteristics: gate and source potentials (with respect to the fixed drain potential).

3.3.1. Gate Potential. Device conductance can be modulated via the gate potential mainly through modulation of the Schottky barrier width. The latter being fixed by the semiconducting material and its doping level in 3D structures, field screening in 1D SWNTs is weak and band bending due to gate potential variations can be used to change the barrier thickness and hence the tunnelling probability. It was shown [37] that thermally assisted tunnelling, and not thermionic emission, dominates carrier injection into $1 \mathrm{D}$ semiconductors. For nicely controllable devices, a variation of $1 \mathrm{~V}$ on the gate potential is sufficient to switch the device from the off- to the on-state $[30,36]$ and sweep through six decades of drain current. Thick oxides can require gate voltage excursions of tens of volts [38]. Although precise values of the derivative of the conductance with respect to the gate potential depend on the exact zero-bias Fermi level position with respect to the valence and conduction bands, as well as on the controllability efficiency, the on/off transition generally happens in the $\pm 5 \mathrm{~V}$ range. Consequently CNFETs show ambipolar characteristics, which can be suppressed via gate structure [39] and contact engineering. Section 5 reveals the optimal bias point for electromechanical operation to lie somewhere between the transition and the on-state.

3.3.2. Source Potential. The drain-source voltage $V_{d s}$ influences the device performance to a lesser extent than the gate potential, but must obey some constraints. A nonzero $V_{d s}$ being necessary to cause charges to flow, the current increases linearly with $V_{d s}$ until saturation occurs. Beyond a critical value of $V_{d s}$, minority carrier injection sets in, leading to nonnegligible off-state currents and an exponential increase in on-current beyond the $25 \mu \mathrm{A}$ saturation limit, with risk of device destruction. A CNFET acts as two Schottky barriers connected via a low-field Drude resistance. Assuming a midgap lineup of the Fermi level with respect to the bandgap and keeping $V_{g}$ close to $V_{s}$ while increasing $V_{d}$ lead to band bending at the drain and enhanced hole injection. Sweeping $V_{g}$ towards $V_{d}$ now causes the drain Schottky barrier for holes to widen and the Schottky barrier width for electrons at the source to shrink. At $V_{g}=\left(V_{d}+V_{s}\right) / 2$, the current through the SWNT becomes minimal, then increases again with electrons as the majority carriers. Thus, larger $V_{d s}$ imply the difficulty to maintain wide Schottky barriers simultaneously for both types of carriers. If proper transistor behaviour is defined via an on/off current ratio of at least $10^{4}$, an upper limit for $V_{d s}$ is given [36] as a function of controllability (oxide thickness $\left.t_{\mathrm{ox}}\right)$ and bandgap (diameter):

$$
V_{d s, \max }[\mathrm{V}]=\left(E_{g}^{1.3}[\mathrm{eV}]-0.2\right) \sqrt{t_{\mathrm{ox}}[\mathrm{nm}]} .
$$

For CNFETs, a reasonable range of $V_{d s}$ spans from $0.01 \mathrm{~V}$ for highly controllable small bandgap designs to some volts for large bandgap designs with reduced controllability.

3.4. Device Operation. Once properly biased, small-signal variations can be superposed on the different terminals to use the CNFET as a capacitor, transistor, or mixer.

3.4.1. Similarities with the FET. The gate voltage's $V_{g}$ efficiency of modulation of the drain current $I_{d}$ is expressed as a transconductance $g_{m}=\partial I_{d} / \partial V_{g}$, reaching peak values of $30 \mu \mathrm{S}[12,26,27]$ at the onset of conduction, and decreases in the saturation regime. The inferred CNFET current modulation is

$$
\delta I_{\mathrm{fet}}^{\omega_{\text {in }}}=g_{m} \cdot \delta V_{g}^{\omega_{\text {in }}}
$$

The electrical FET current has the same frequency $\omega_{\text {in }}$ (indicated by superscript notation throughout this paper) as the driving voltage and can take values up to $\mu \mathrm{A}$ in highly controllable geometries. As the current is mainly controlled via the Schottky barrier widths, CNFETs can be controlled equivalently through the gate and source (for electrons as majority carriers) or drain (for holes as majority carriers). Average transconductances of $1.5 \mu \mathrm{S}$ for long and $12 \mu \mathrm{S}$ for short devices were found [12] to be independent of frequency. With gate-drain capacitances of $\sim 100 \mathrm{aF}$, such transconductances lead to state-of-the-art unity-gain frequencies of $50 \mathrm{GHz}$ [12] imposed by $f_{T}=g_{m, e l} / 2 \pi C_{g d}$. To read the $\mathrm{GHz}$ operation with these transconductances, the tracks must be of sufficiently low resistance and minimal capacitance to the substrate and other signals, to avoid low-pass filtering of the signal. The critical track $\mathrm{RC}$ product $\mathrm{RC}_{\text {track }}=1 / 2 \pi f_{T} \approx$ 100 ps requires very careful signal routing. Suspension of the channel sacrifices part of this performance and the lesser controllability leads to typical transconductances of tens of $\mathrm{nS}$, resulting in current amplitudes up to tens of $\mathrm{nA}$.

3.4.2. Signal Mixing. CNFETs can also be driven simultaneously from the source and the gate and hence be operated as microwave mixers. As readout happens at lower frequencies, the corresponding track design is less crucial and the resulting low frequency current writes

$$
I_{\text {mix }}^{\Delta \omega}=\frac{1}{2} g_{m} \delta V_{s}^{\omega_{\text {in }}-\Delta \omega} \delta V_{g}^{\omega_{\text {in }}}
$$

and has been measured up to mixing signals of $10 \mathrm{GHz}$ [40]. The theoretical upper limit, given by the quantum capacitance $\left(C_{d}=C_{\text {quant }}\right)$, predicts unity gain to scrape terahertz [41].

3.4.3. Capacitive Feedthrough and Miller Effect. Figure 1 suggests that any ac-signal capacitively bridges gate and drain. The coupling capacitance $C_{\mathrm{G}-\mathrm{CNT}}$ comprises the intrinsic 
device gate-drain capacitance $(\sim 100 \mathrm{aF})$ and the track-totrack capacitances, which might contribute up to femtofarads. The resulting gate-induced current modulation in the drain can easily reach $\mu \mathrm{A}$ amplitudes at $\mathrm{GHz}$.

$$
\delta I_{\text {cap }}^{\omega_{\text {in }}}=C_{\mathrm{G}-\mathrm{CNT}} \omega_{\text {in }} \delta V_{g}^{\omega_{\text {in }}} .
$$

This capacitance not only feeds forward part of the signal without amplification by the FET effect, but also has even a Miller effect on the gate signal. Multiplied by the voltage gain of the stage, this capacitance must be minimized to prevent a severe degradation of the maximum intrinsic device frequency.

Before projecting the NEMS into the purely mechanical domain to evaluate its dynamics, let us put on record the NEMS design strategy: proper tube selection $(L=0.1-1 \mu \mathrm{m}$, $d=2-5 \mathrm{~nm}$ ), device (Al, Cr or Pd electrodes, minimal gatedrain coupling), and readout (minimal $\mathrm{RC}_{\text {track }}$ ) design, along with appropriate bias $\left(V_{d s}=0.01-1 \mathrm{~V}, V_{g}=-5 \cdots+5 \mathrm{~V}\right.$ inside the transition/on-state, $I_{\text {on }}=1 \mu \mathrm{A}$ ). Orders of magnitude of the strength and frequency of the three purely electrical contributions to the drain current are summarized in Table 1, indicating that capacitive feed-through starts masking the transistor effect at $\mathrm{GHz}$ frequencies and higher.

\section{Mechanical Properties of CNT Resonators}

Proper model selection is the key to accurate results. It has been shown [42] that nonlinear continuum models yield good match with the more complicated molecular dynamics models. We here describe the CNT by an Euler-Bernoulli beam model that accounts for the geometric nonlinearity, but neither buckling nor slack. Slack is anyhow an obstacle to high quality resonance and can be eliminated by prestraining the tube. The partial differential equation of motion, in terms of Young's modulus $E$, areal moment of inertia $I$, crosssectional area $A$, stress at rest $s_{0}$, and damping coefficient $c$

$$
\begin{aligned}
F_{\mathrm{ext}}= & E I \frac{\partial^{4} w}{\partial x^{4}}+\rho A \frac{\partial^{2} w}{\partial t^{2}}+c \frac{\partial w}{\partial t} \\
& -\left(s_{0} A+\frac{E A}{2 L} \int_{0}^{L}\left(\frac{\partial w}{\partial x}\right)^{2} d x\right) \frac{\partial^{2} w}{\partial x^{2}}
\end{aligned}
$$

expresses the CNT's displacement $w(x)$ caused by an external force $F_{\text {ext }}[\mathrm{N} / \mathrm{m}]$. The first two terms describe the equilibrium between strain and kinetic energy, followed by the damping term and the geometric nonlinearity due to mid-plane stretching. The response to an external force (6) is solved in the clamped-clamped configuration via a reduced-order model based on the Galerkin procedure, which is a good compromise between finite elements and a lumped model.

4.1. CNT Properties and Device Design. The discussion will be limited to the design-parameter set, whose values the designer can influence, such as tube length and diameter. Large young moduli and mechanical quality factors of defectfree tubes are favoured.
TABLE 1: Expected orders of magnitude of the contributions to the drain current (for $\delta V_{g}^{\omega_{\text {in }}}=1 \mathrm{~V}$ ).

\begin{tabular}{lccc}
\hline Effect & Information & Frequency & Amplitude (A) \\
\hline Mixer & Electrical & $\Delta \omega$ & $10^{-7}$ \\
Mixer & Motional & $\left|\omega_{0}-\omega_{\text {in }}-\Delta \omega\right|$ & $10^{-9}$ \\
Fet & Electrical & $\omega_{\text {in }}$ & $10^{-7}$ \\
Capacitive & Electrical & $\omega_{\text {in }}$ & $10^{-15} \omega_{\text {in }}$ \\
Fet & Motional & $\omega_{0}$ & $10^{-9}$ \\
Piezo & Motional & $\omega_{0}$ or $2 \omega_{0}$ & $10^{-8}$ \\
\hline
\end{tabular}

4.1.1. Static Behaviour. Figure 3 provides insight into the steady force, homogeneously distributed along the tube length, necessary to deflect the CNT transversally. Midplane stretching translates into a nonconstant stiffness and the forces may span several orders of magnitude as a function of the tube's diameter. With small- and medium-diameter tubes being the most interesting for electromechanical applications (1), forces of tens of $\mathrm{nN}$ will always push the tube to its elastic limits of roughly $5 \%$ strain [43]. Contrariwise, a minimal force is required to overcome the incoherent sum of all stochastic processes driving the resonator. By the fluctuationdissipation theorem and regardless of the origin of the dissipation mechanism, the motion of the NEMS ultimately thermalizes into heat. Given that quantum fluctuations are negligible at ambient temperature and radio-frequencies $\left(k_{B} T \gg \hbar \omega_{0}\right)$ [44], the classical equipartition law predicts an average energy of $k_{B} T$ per mode, with $T$ being the physical temperature of the NEMS. This established thermomechanical noise energy [45] may infer an upper bound on the thermal fluctuations $w_{x}$ along the tube. Hypothesizing a homogeneously distributed force, the fluctuations are implicitly defined by the system's energy or explicitly by its coenergy:

$$
\begin{aligned}
k T & =\left\langle E_{x}\right\rangle=\left\langle\int_{z=0}^{w_{x}} F_{x}(z) \mathrm{d} z\right\rangle \\
& =F\left\langle w_{x}(F)\right\rangle-\int_{f=0}^{F}\left\langle w_{x}(f)\right\rangle \mathrm{d} f .
\end{aligned}
$$

This thermomechanical noise energy is reported in Figure 3 and illustrates the narrow linear dynamic range of high aspect-ratio tubes [46]. Similar displacements can be reached by driving the tube harmonically at its resonance frequency, with a force that, in the linear regime, is $Q$ times smaller, with $Q$ being the mechanical quality factor. Although quality factors of $10^{5}$ have been observed at cryogenic temperatures [47], ambient temperature reduces them to about 100 $[5]$.

4.1.2. Dynamic Behaviour. The afore mentioned fundamental resonance frequency is predicted by the Euler-Bernoulli model to scale as

$$
f_{\text {res }}=\frac{1}{\sqrt{3 \rho}} \sqrt{E \pi^{2} \frac{r^{2}}{L^{4}}+s_{0} \frac{1}{L^{2}}},
$$




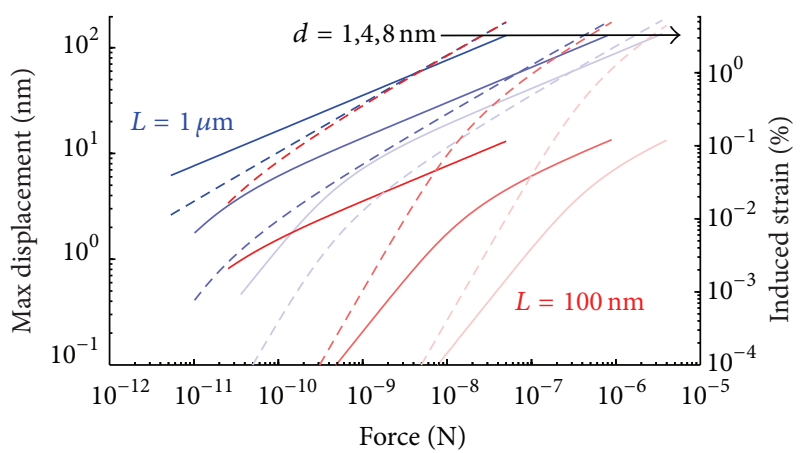

FIGURE 3: Force-displacement (continuous line) and force-strain (dashed line) relation for suspended, nonprestrained CNTs within the elastic limit and above the thermomechanical noise floor $(300 \mathrm{~K})$.

where $r$ is the radius, $L$ is the length, and $\rho$ is the density of the CNT. As can be concluded from Figure 4 , the mere strain induced during oscillation might be sufficient to stiffen the tube and increase its resonance frequency, leading the linear prediction into considerable error. The dynamic behaviour of high aspect ratio tubes is once again shown to be severely confined by the thermomechanical noise and the onset of the nonlinear regime.

4.2. Mechanical Tuning. Controllability of the source position opens the option to prestrain the tube. The induced strain relates to the applied force via the stress-strain curve, with its linear regime expression being

$$
s_{0}=\frac{\Delta L}{L}=\frac{1}{E \pi r^{2}} F
$$

Although such a straining technique might be slightly less efficient from a force-strain perspective, its advantage is twofold. In contrary to the transversal force [5], this longitudinal force can be applied mechanically [8] during resonance, meaning that higher forces are available with no impact on the electronic terminal potentials, setting the electronic operation regime, highly sensitive to the bias. Additionally, slack can be compensated by pulling the tube till the onset of strain while preserving symmetric oscillation (Section 6).

\subsubsection{Resonance Frequency Tuning and Linearization.} Figure 5 illustrates what formula (8) predicts. By prestraining the tube sufficiently, the resonance frequency can be tuned over a couple of decades and turns independent of the tube's diameter. Note that the force necessary to induce this prestraining remains very well a function of the diameter (9). The designer has to trade off between the wider tuning ranges of long $(10 \mathrm{MHz}-1 \mathrm{GHz})$ tubes and the larger linear dynamic range of short $(1 \mathrm{GHz}-10 \mathrm{GHz})$ tubes. Besides frequency tuning, prestraining also allows to weaken the resonance frequency's sensitivity to the oscillation amplitude, enabling trivial oscillation start-up at system level.

4.2.2. Tube Stiffening. Although, this tuning option might look tempting, designers should keep in mind that the

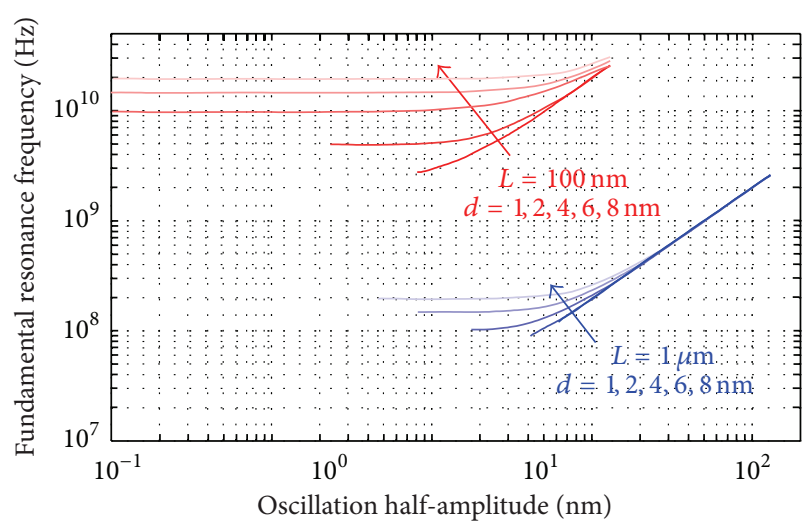

FIGURE 4: Resonance frequency for nonprestrained CNTs with oscillation amplitudes from thermomechanical noise floor up to the maximum strain limit (5\%). The onset of mid-plane stretching translates into increasing resonance frequencies.

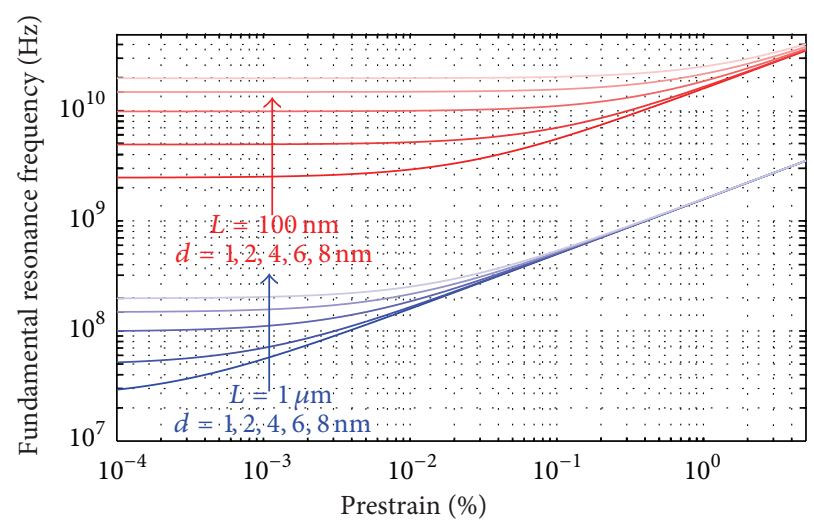

FIGURE 5: The fundamental resonance frequency as a function of prestrain is tunable over $1(L=100 \mathrm{~nm})$ or $2(L=1 \mu \mathrm{m})$ decades.

pulling force on the tube has to be increased appropriately to overcome the prestraining force and deflect the tube, as depicted in Figure 6. The thermo-mechanical fluctuations are reduced by the same principle. It is exactly this demand for high force that will limit the tuning of tubes (Section 5).

The presented force-displacement relations for electrically interesting tubes (1) reveal that the necessary driving forces span a wide range from $p N / Q$ to $\mu N / Q$. The forcestrain relations impact the detection mechanisms, studied in Section 5. Linear resonance frequencies range from tens of $\mathrm{MHz}(L=1 \mu \mathrm{m})$ to tens of $\mathrm{GHz}(L=100 \mathrm{~nm})$ and tube straining allows for tuning over a couple of decades along with an increase of the linear dynamic range. This feature comes at the expense of larger minimal driving forces (see Figure 6).

\section{Carbon Nanotube Electromechanical Resonators}

For the CNT to serve as NEMS and the circuit to read motional information, the signal has to flow from the backend's electrical to the NEMS' mechanical back into the 


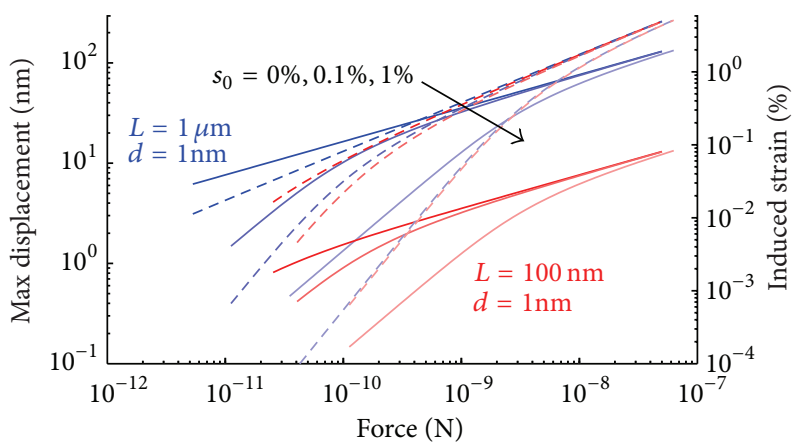

FIGURE 6: Force-displacement (continuous line) and force-strain (dashed line) relation for suspended, prestrained CNTs within the elastic limit and above the thermomechanical noise floor $(300 \mathrm{~K})$.

frontend's electrical domain. Any shortcut bypassing the mechanical world constitutes an undesirable parasitical feedthrough.

5.1. Actuation. The roots of actuation lie in the two-port capacitor, formed by the gate electrode and the CNT itself (Figure 2). As the energy stored in this capacitor can be modulated via the charge in the electrical domain and via the gate-CNT distance in the mechanical domain, transdomain signal flow becomes possible. Neglecting the contribution of the density of states in the CNT and approximating the device as a long equipotential cylinder above an infinite plate, the gate-CNT capacitance writes

$$
C_{\mathrm{G}-\mathrm{CNT}}=\int_{0}^{L} \frac{2 \pi \epsilon}{\operatorname{acosh}((h-w(x)) / r)} \mathrm{d} x,
$$

where $w(x)$ is the transversal motion as a function of the position along the tube and $\epsilon$ is the gap permittivity. As we are controlling the voltage $V$ rather than the charge, our reasoning shall be based on the coenergy $W^{*}(V, w)=C V^{2} / 2$, entailing the expression of the force on the tube, suspended at height $h$ over the gate electrode:

$$
\begin{aligned}
F & =-\frac{\partial W^{*}(V, w)}{\partial w} \\
& =\int_{0}^{L} \frac{\pi \epsilon V^{2}}{\sqrt{h-w} \sqrt{h-w+2 r} \cdot \operatorname{acosh}^{2}((h+r-w) / r)} \mathrm{d} x .
\end{aligned}
$$

5.1.1. Electrostatic Force Amplitude. For small oscillation amplitude $(w(x) \ll h)$ and to first order, this force scales with the device geometry as $L / h$, with $L$ being the tube suspension length and $h$ its distance to the gate electrode. This force is relatively insensitive to the tube radius $r$ (supposing $h \gg r$ ). Formula (12) provides a good estimate on the effective force pulling on the SWNT:

$$
\begin{aligned}
& \log _{10}\left(F_{\text {eq }}\right) \\
& = \begin{cases}-12+\log _{10}\left(\frac{L}{h}\right)+2 \log _{10}(V)+\log _{10}(Q), & @ \omega_{0} \\
-12+\log _{10}\left(\frac{L}{h}\right)+2 \log _{10}(\mathrm{~V}), & \text { else. }\end{cases}
\end{aligned}
$$

Energy conservation at resonance leads to larger apparent forces compared to the static case. Figures 3 and 6 translate the equivalent mechanical force $F_{\text {eq }}$ directly into an oscillation amplitude and the related induced strain. At room temperature and depending on the prestrain, a pristine tube $(Q=100)$ in a good setup $(L / h=10)$ would require a voltage amplitude of $0.1 \mathrm{~V}$ to $1 \mathrm{~V}$ to sustain a $10^{-5}$ to $10^{-3}$ strain variation at resonance. Larger driving voltages are not acceptable, as they firstly would impact the electrical operating point, eventually switching the device from on- to off-state, and secondly might increase the electrostatic force to a value that the elastic restoring force can no longer resist, leading to a sudden collapse of the structure, named dynamic pull-in [48].

5.1.2. Electrostatic Force Frequency. Decomposing the driving voltage into a continuous $V_{g}^{0}$ and an harmonic $V_{g}^{\omega} \cos (\omega t)$ excitation reveals that the force, proportional to the voltage squared, possesses three frequency components, at $0, \omega$, and $2 \omega:$

$$
\begin{aligned}
F_{\text {eq }} \sim & \left(\left(V_{g}^{0}\right)^{2}+\frac{1}{2}\left(V_{g}^{\omega}\right)^{2}\right)+\left(2 V_{g}^{0} V_{g}^{\omega}\right) \cdot \cos (\omega t) \\
& +\left(\frac{1}{2}\left(V_{g}^{\omega}\right)^{2}\right) \cdot \cos (2 \omega t) .
\end{aligned}
$$

If there is no accumulated charge $\left(V_{g}^{0}=0\right)$ or this charge is trapped in defect states, the electrostatic force only displays a $2 \omega$ component in the Fourier spectrum. For the device to be in the on-state, a nonzero $V_{D C}$ might nevertheless be indispensable, leading to an increasingly strong $1 \omega$ response, assuming that the excess charges have sufficient mobility to follow the RF gate signal. Consequently, to excite the CNT's mechanical resonance at $\omega_{0}$, the necessary gate driving frequency must be

$$
\omega_{\text {in }}= \begin{cases}\omega_{0}, & \text { with excess charges } \\ \frac{\omega_{0}}{2}, & \text { without excess charges. }\end{cases}
$$

5.2. Motion Detection. The motional information can be inferred via two different physical phenomena. One is the CNT's conductance in a potential field, and the other is the piezoresistive property of CNTs. For the investigation of both detection mechanisms, we suppose the tube to be vibrating harmonically at a frequency $\omega_{0}$, while it is driven at a frequency $\omega_{\text {in }}$.

5.2.1. Field Effect. The channel motion in a potential field modulates the Schottky barrier width and the charge induced on the tube. By the fact that the conductance change for semiconducting [9] and small-bandgap [49] SWNTs is proportional to the charge variation on the tube, the motion in a changing potential field influences the conductance $G$ as

$$
\delta G=\frac{g_{m}}{V_{D S}}\left(\delta V_{g}^{\omega_{\mathrm{in}}}+\frac{V_{g}}{C_{g}} \delta C_{g}^{\omega_{0}}\right) .
$$


Electrical Field Effect. The conductance change due to the gate voltage variation is the purely electrical field effect analyzed in Section 3.4, entailing a parasitical feed-through expressed by (3) of tens of $\mathrm{nA}$ at the driving frequency $\omega_{\text {in }}$.

Motional Field effect. The useful component of the field effect originates from the displacement $\delta z$ of the tube, yielding a current, smaller than its electrical counterpart $\delta I_{\text {fet }}^{\omega_{\text {in }}}$,

$$
\delta I_{\mathrm{fet}}^{\omega_{0}}=\frac{g_{m} V_{G}}{\sqrt{h-w} \sqrt{h-w+2 r} \cdot a \cosh ((h+r-w) / r)} \delta z,
$$

and not exceeding the $\mathrm{nA}$ floor at the vibrating frequency. This is subjected to the condition that the gate potential, originally reserved for biasing, creates the required potential field.

5.2.2. Piezoresistivity. The piezoresistive transduction principle can be traced back to the bandgap sensitivity of CNTs to strain. While axial strain moves the K-points of the rolledup graphene sheet, the Poisson ratio caters for a reduction in the tube diameter and new boundary conditions, entailing a strain-dependent bandgap. This strain dependence is most pronounced in metallic zig-zag SWNTs, while totally absent in metallic armchair SWNTs. All other chiralities find their bandgap sensitivity to a strain $\varepsilon$ between those two extremes, with maximum sensitivities $d E_{g} / d \varepsilon$ predicted to reach up to $100 \mathrm{meV} / \%$, depending on the model [50-53]. This quasi omnipresence turns piezoresistive component detection into a reliable readout strategy. Besides contact strain, modulating the tunnelling barrier width and hence contact resistance, the induced strain can be sensed indirectly through the change in resistance it causes, characterized through the gauge factor $\mathrm{GF}=(\Delta R / R)(1 / \varepsilon)$. With thermally activated transport being most sensitive on the bandgap, the largest GFs are measured in the device off-state, where transport is dominated by exactly this phenomenon. At symmetrical oscillation around the tube's rest position, the piezoresistive current has twice the frequency of the mechanical vibration, while their frequency is identical otherwise,

$$
\delta I_{\text {piezo }}^{2 \omega_{0} \mid \omega_{0}}=\mathrm{GFE}_{d}^{0}
$$

and reveals the existence of an optimal bias point. While the off-state is favourable to large GF, acceptable drain bias current $I_{d}^{0}$ requires the device to be in the on-state. The tradeoff lies in the transition state, close to the maximum transconductance bias. Prestraining was shown to enhance the GF from 856 to 2900 [54] and the larger off-currents of large-diameter tubes $(d>2 \mathrm{~nm})$ are beneficial. It was shown that GFs of 100 may coexist with bias currents of $1 \mu \mathrm{A}$ in small-gap semiconducting SWNTs [55], leading to piezoresistive currents of 1 to $100 \mathrm{nA}$ in the targeted $10^{-5}$ to $10^{-3}$ strain region.
5.3. Parasitical Feed-Through. Finally, the parasitical feedthrough from the driving electrode, analyzed in Section 3.4, and scaling with frequency

$$
\delta I_{\text {cap }}^{\omega_{\text {in }}}=C_{\mathrm{G}-\mathrm{CNT}} \omega_{\text {in }} \delta V_{g}^{\omega_{\text {in }}}
$$

comes with a strength overshadowing the motional components at frequencies higher than $100 \mathrm{MHz}$. While device and track designs minimizing the gate-drain capacitive coupling are an asset, the only loophole lies in frequency separation and signal filtering, as will be discussed subsequently.

Among all contributions to the drain current, the motional field effect $\left(\omega_{0}\right)$ and the piezoresistive component $\left(\omega_{0}\right.$ or $\left.2 \omega_{0}\right)$ were shown to encode information on the CNT's motion. In accordance with [6], we find the quasi omnipresent piezoresistive response more pronounced than the motional field effect. The electrical field effect $\left(\omega_{\text {in }}\right)$ and capacitive coupling $\left(\omega_{\text {in }}\right)$ on the other hand constitute the parasitical feed-through. With the latter dominating the output signal at frequencies above hundreds of $\mathrm{MHz}$, it is essential to select the useful frequencies via filtering. Table 1 summarizes orders of magnitude of the strength and frequency of these signals.

\section{Open-Loop Resonators}

The NEMS' electromechanical characterisation uses to happen in a laboratory context allowing the rich use of sophisticated equipment such as high magnetic fields [56], optical interferometry [57], spectrum- and network analyzers. Timeaveraging and mixing techniques seem to be the trend for CNT-NEMS resonator characterisation. This section reviews the most common techniques, analyzes from which draincurrent component (Table 1) they infer motional information, and reveals the existence of a yet unexploited $4 \omega$ mixing technique.

6.1. Time-Averaging. Time-averaging techniques stimulate the NEMS with a slowly varying frequency ramp at the gate, while a constant DC bias fixes the source and drain potentials. Upon motion, the drain current is instantly modulated, and if it has a nonlinear dependence on the gate voltage, the variation does not cancel out over one oscillation, meaning that the average drain-current holds precious information about the oscillation amplitude. A short-term integration of the drain-current allows thus to detect resonance, based on nonlinear piezoresistive [4] or electrostatic interactions in the vicinity of Coulomb oscillations within quantum dots [47]. Simultaneously, purely electrical contributions to the drain current must either react linearly to the gate voltage or be frequency independent [12].

6.2. Signal Mixing. Similar to its electrical counterpart (Section 3.4), the device can also be operated as an electromechanical mixer. For this purpose, the source terminal is driven at a frequency $n \cdot \omega_{\text {in }} \pm \Delta \omega$, while the gate is driven at $\omega_{\text {in }}$. In such a mixing setup, the gate signal defines the actuation, while the source signal selects the drain-current contribution to be detected via lock-in at $\Delta \omega$. 
Defining the CNT's resonance frequency as $\omega_{0}$, equation (14) leads to resonance for an $\omega_{\text {in }}=\omega_{0} / 2$ gate frequency in the absence of RF modulable static charges and for a $\omega_{\text {in }}=\omega_{0}$ actuation if such charges invade the CNT. Device bias and the presence or absence of mechanical prestrain may force the CNT to oscillate symmetrically or asymmetrically around its least-strain position, with the former causing the CNT to bend twice per oscillation cycle, yielding a piezoresistive drain-current contribution at twice the oscillation frequency. Table 2 highlights the different drain-current contributions' frequency for each of these four possible situations. The signals' strength can be read from Table 1 . Three values for the ratio $n$ between indirect detection and actuation frequency allow to read motional information, of which two have been successfully tested and reported in the literature so far: the $1 \omega$ [10] and the $2 \omega$ [6] techniques. We here point out that a nevermentioned $4 \omega$ technique exists, which detects CNT motion via the piezoresistive contribution, allows to determine the resonance frequency uniquely, and further separates the motional from the parasitical information by a factor four in frequency. The full advantages of this new technique will stick out in closed-loop topologies (Section 7). While it seems tempting to shift the signal to low frequencies in order to circumvent the afore mentioned high-frequency obstacles, the price to pay is phase information loss and consequently the impossibility to operate the NEMS in a self-regulating closed-loop configuration.

\section{Closed-Loop Oscillators}

The way towards future closed-loop operation poses two challenges. On one hand the motional information must be isolated from the parasitical one, while on the other hand, the signal must be detectable from the background noise.

7.1. Spectral Separation. Although the electrical contributions to the drain current overwhelm the motional ones, the $1 \omega$ mixing technique allowed to detect resonance by the mere fact that the motional information is frequency dependent, while the electrical contributions depend relatively less or not at all on frequency [12]. Using this small variation in a locally steady large signal for closed-loop self-regulation seems not straight-forward. The $2 \omega$ approach is extendable to closedloop topologies in the sense that a factor two, in terms of frequency, separates the motional from the parasitical information. To infer the mechanical resonance frequency from the motional information uniquely, quantitative knowledge of static charges or oscillation symmetry is indispensable. The $4 \omega$ technique would separate motional and parasitical information further and hence require less aggressive filtering. Also the mechanical frequency can be inferred uniquely. The disadvantage of this approach remains to be the requirement for symmetrical oscillation at the absence of static charges, which may or may not be compatible with acceptable device bias (Section 3.3), depending on the device. To transform the 1:100 ratio between the motional and parasitical signals at $\mathrm{GHz}$ frequencies into a $10: 1$ proportion, a 10th-order Butterworth or a 6th-order Chebyshev band-pass filter is required in the $2 \omega$ case, while 5 th and 4 th order are, respectively, necessary in the $4 \omega$ case.
TABLE 2: Spectral components of the drain-current, with or without static charges $\left(q_{0}, \bar{q}_{0}\right)$ and symmetrical or asymmetrical $\left(s_{0}, \bar{s}_{0}\right)$ oscillation, as detected by the $1 \omega, 2 \omega$ and $4 \omega$ mixing techniques.

\begin{tabular}{cccccccc}
\hline & \multicolumn{2}{c}{ Motional } & \multicolumn{2}{c}{ Electrical } & \multicolumn{3}{c}{ Detected } \\
& FET & Piezo & Fet & Capacitive & $1 \omega$ & $2 \omega$ & $4 \omega$ \\
\hline $\bar{q}_{0}, \bar{s}_{0}$ & $\omega_{0}$ & $\omega_{0}$ & $\omega_{0} / 2$ & $\omega_{0} / 2$ & $\omega_{0} / 2$ & $\omega_{0}$ & - \\
$\bar{q}_{0}, s_{0}$ & $\omega_{0}$ & $2 \omega_{0}$ & $\omega_{0} / 2$ & $\omega_{0} / 2$ & $\omega_{0} / 2$ & $\omega_{0}$ & $2 \omega_{0}$ \\
$q_{0}, \bar{s}_{0}$ & $\omega_{0}$ & $\omega_{0}$ & $\omega_{0}$ & $\omega_{0}$ & $\omega_{0}$ & - & - \\
$q_{0}, s_{0}$ & $\omega_{0}$ & $2 \omega_{0}$ & $\omega_{0}$ & $\omega_{0}$ & $\omega_{0}$ & $2 \omega_{0}$ & - \\
\hline
\end{tabular}

7.2. Minimum Detectable Signal. To conclude this walkthrough of CNT NEMS operation within electronic circuits, let us finally assess the ability of standard electronic devices, such as bipolar junction (BJT) or field effect (FET) transistors, to sense and amplify the currents reported in Table 1. Supposing displacements exceeding the thermal fluctuations (Section 4) and device operation in the $\mathrm{MHz}$ to $\mathrm{GHz}$ band, above the corner frequency [58], where white noise dominates, the minimum detectable signal (MDS) depends on the signal-to-noise ratio (SNR) necessary for subsequent signal processing, the frontend's noise figure $(\mathrm{NF})$, and the circuit's bandwidth $B$, defined by the bandpass filter (see Figure 1):

$$
I_{\mathrm{CNT}, \mathrm{RMS}} \geq \sqrt{\frac{4 k T B}{R_{\mathrm{CNT}}} \cdot 10^{\mathrm{NF}} \cdot \mathrm{SNR}_{\mathrm{out}}}
$$

With the frontend being a cascade of stages, its noise figure is expressed via Friis' formula:

$$
\mathrm{NF}_{\text {total }}=\log _{10}\left(F_{1}+\sum_{i=2}^{n} \frac{F_{i}-1}{\prod_{j=1}^{i-1} G_{j}}\right),
$$

where $F_{i}$ and $G_{i}$ denote the noise factor and power gain of stage $i$. $\mathrm{NF}_{\text {total }}$ shall be minimized under the constraint of overall sufficient gain. The resulting MDS shall be lower or equal to the signal provided by the CNT as reported in Table 1. Considered candidate circuits operate the BFP750, a high linearity low noise Silicon-Germanium-Carbon NPN transistor, in a common emitter (CE) configuration. We make the reasonable assumption that bias resistors exceed the transistor's base impedance up to $\mathrm{GHz}$ frequencies, making their noise contributions negligible. The CNT is interfaced by the discrete component frontend [59], as shown by the inset of Figure 7, which drives an integrated signalprocessing CMOS feed-back loop [60]. Hence, the interface capacitances are of the order of $C=1 \mathrm{pF}$ [59]. The frontend's transimpedance writes

$$
A_{\Omega, \mathrm{CE}}=\frac{1}{1+s h_{\mathrm{fe}, 1} \cdot C / g_{m 1}} \cdot \prod_{i=1}^{n} h_{\mathrm{fe}, i} \cdot \frac{1}{s C}
$$

and must be able to convert a $\ln \mathrm{A}$ current variation into a $10 \mathrm{mV}$ stimulus for the CMOS IC, hence, exceeding $10^{7} \Omega$. 


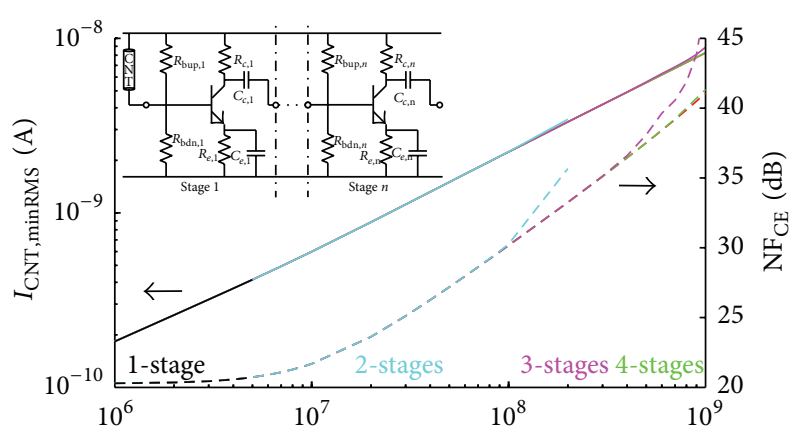

FigURE 7: Gain-constrained minimum detectable signal and NF as a function of frequency and number of stages for $\mathrm{CE}$ frontend and $\mathrm{SNR}_{\text {out }}=1$.

Given the CNT's thermal current noise density $4 k T / R_{\mathrm{CNT}}$ and the transistors' base $2 q I_{B}$

$$
\mathrm{NF}_{\mathrm{CE}}=\log _{10}\left(1+\frac{R_{\mathrm{CNT}}}{\left|1+s h_{\mathrm{fe}, 1} \cdot C / g_{m 1}\right|} \cdot \sum_{i=1}^{n} \frac{g_{m, i}}{\prod_{j=1}^{i} h_{\mathrm{fe}, j}}\right) .
$$

Completed by further constraints for acceptable transistor bias ( $1 \mathrm{~mA}$ to $100 \mathrm{~mA}$ ), the constrained optimization problem is solved via sequential quadratic programming (SQP) and leads to Figure 7. The strikingly high NF is entirely ascribed to the NEMS' high output impedance in combination with a picofarad interconnect capacitance. Practice shows that all but $1 \mathrm{~dB}$ come from the most noise-critical first stage. While a single stage can provide the necessary gain up to few $\mathrm{MHz}$, cascading is necessary for higher frequencies. Although Figure 2 stages work out up to $200 \mathrm{MHz}$, the use of 3 stages allows to relax the gain constraint and provides notably better noise figures. Close to optimal noise figures can be achieved by 4 stages up to $\mathrm{GHz}$. The optimal bias, with sufficient number of stages to ensure gain, lies at the lower bound of $1 \mathrm{~mA}$ for the BFP750. Combining this with the fact that most of the SNR degradation is due to the first stage, the addition of supplementary stages does not compromise the NF and an optimally biased 4-stage frontend will perform nearly optimal for any signal frequency from $\mathrm{MHz}$ to $\mathrm{GHz}$. Requiring an output SNR of 10 at $1 \mathrm{GHz}$, Figure 7 indicates that the CNT signal must exceed $25 \mathrm{nA}$, which is probably not the case (see Table 1). Bandpass filtering, which is limited to filter quality factors below the NEMS's quality factor ( 100 at room temperature), if CNT-based oscillator applications as in Figure 1 are targeted, may relax this constraint to $2.5 \mathrm{nA}$ $\left(Q_{\text {filter }}=100\right)$ and allow a 4-stage BFP750 common emitter frontend to sense CNT motion up to $\mathrm{GHz}$ without phase information loss. This filter must follow the frontend, but preliminary filters for spectral separation (see Section 7.1) can interlace the frontend's stages, given the negligible SNR degradation due to all but the first stage.

\section{Conclusion}

In the scope of combining the carbon nanotube NEMS' ability of fine mechanical sensing with the vast functionalities available in electronic circuit design, synthetic guidelines on proper carbon nanotube selection, NEMS resonator, and readout design, along with appropriate bias and operation, are at the outcome of an analysis of state-of-the-art results in the respective domains. Orders of magnitude of electrical and mechanical components forming the NEMS output spectrum were extracted and tabulated. Motion inference from the piezoresistive current contribution was shown to be the most reliable and a new $4 \omega$-approach henceforth completes the set of readout techniques. Signal isolation from parasitical feedthrough and background noise has been shown to be possible via cascaded amplification without phase information loss and up to $\mathrm{GHz}$ frequencies. This fact entails promises for highly functional, tunable, and sensitive systems emerging from the combination of carbon nanotube NEMS with the established CMOS integrated circuits.

\section{Acknowledgments}

This research is funded by Nano-Tera.ch and evaluated by SNSF.

\section{References}

[1] L. Radushkevich and V. Lukyanovich, "About the structure of carbon formed by thermal decomposition of carbon monoxide on iron substrate," Zhurnal Fizicheskoi Khimii, vol. 26, pp. 8895, 1952.

[2] S. Iijima, "Helical microtubules of graphitic carbon," Nature, vol. 354, no. 6348, pp. 56-58, 1991.

[3] C. Kauth, M. Pastre, and M. Kayal, "On-chip mass sensing at the physical limits of nanoelectromechanical systems," in Proceedings of the Advances in Sensors and Interfaces, pp. 131-135, Bari, Italy, June 2013.

[4] H. Chandrahalim, C. I. Roman, and C. Hierold, "Analytic modeling and piezoresistive detection theory of acoustic resonances in carbon nanotubes," in Proceedings of the 10th IEEE Conference on Nanotechnology, (NANO '10), pp. 778-781, Seoul, Republic of Korea, August 2010.

[5] V. Sazonova, Y. Yalsh, I. Üstünel, D. Roundy, T. A. Arlas, and P. L. McEuen, "A tunable carbon nanotube electrochemical oscillator," Nature, vol. 431, no. 7006, pp. 284-287, 2004.

[6] H. B. Peng, C. W. Chang, S. Aloni, T. D. Yuzvinsky, and A. Zettl, "Ultrahigh frequency nanotube resonators," Physical Review Letters, vol. 97, no. 8, Article ID 087203, 2006.

[7] B. Peng, L. Ding, and Z. Guo, "Resonant modelling of two types of tunable carbon nanotube electromechanical oscillators," Micro and Nano Letters, vol. 5, no. 6, pp. 365-369, 2010.

[8] M. Muoth, S. W. Lee, and C. Hierold, "Platform for strainable, TEM-compatible, MEMS-embedded carbon nanotube transistors," in Proceedings of the 24th IEEE International Conference on Micro Electromechanical Systems (MEMS '11), pp. 83-86, Cancún, Mexico, January 2011.

[9] S. J. Tans, A. R. M. Verschueren, and C. Dekker, "Room-temperature transistor based on a single carbon nanotube," Nature, vol. 393 , no. 6680 , pp. 49-52, 1998. 
[10] P. L. McEuen, M. S. Fuhrer, and H. Park, "Single-walled carbon nanotube electronics," IEEE Transactions on Nanotechnology, vol. 1, no. 1, pp. 78-84, 2002.

[11] A. Javey, J. Guo, Q. Wang, M. Lundstrom, and H. Dai, "Ballistic carbon nanotube field-effect transistors," Nature, vol. 424, no. 6949, pp. 654-657, 2003.

[12] J. Chaste, L. Lechner, P. Morfin et al., "Single carbon nanotube transistor at GHz frequency," Nano Letters, vol. 8, no. 2, pp. 525$528,2008$.

[13] P. Burke, "An rf circuit model for carbon nanotubes," in Proceedings of the 2nd IEEE-NANO Conference, pp. 393-396, 2002.

[14] K. Koziol, C. Ducati, and A. H. Windle, "Carbon nanotubes with catalyst controlled chiral angle," Chemistry of Materials, vol. 22, no. 17, pp. 4904-4911, 2010.

[15] H. Guo, Z. Bo, P. Banghua et al., "Direct growth of semiconducting single-walled carbon nanotube array," Journal of the American Chemical Society, vol. 131, no. 41, pp. 14642-14643, 2009.

[16] S. Reich, J. Maultzsch, C. Thomsen, and P. Ordejón, “Tightbinding description of graphene," Physical Review B, vol. 66, no. 3, 2002 .

[17] A. Kleiner and S. Eggert, "Band gaps of primary metallic carbon nanotubes," Physical Review B, vol. 63, no. 7, Article ID 073408, 4 pages, 2001.

[18] W. Song, C. Jeon, Y. S. Kim et al., "Synthesis of bandgapcontrolled semiconducting single-walled carbon nanotubes," ACS Nano, vol. 4, no. 2, pp. 1012-1018, 2010.

[19] C. L. Kane and E. J. Mele, "Size, shape, and low energy electronic structure of carbon nanotubes," Physical Review Letters, vol. 78, no. 10, pp. 1932-1935, 1997.

[20] S. Datta, Electronic Transport in Mesoscopic Systems, Cambridge University Press, Cambridge, UK, May 1997.

[21] J.-C. Charlier, X. Blase, and S. Roche, "Electronic and transport properties of nanotubes," Reviews of Modern Physics, vol. 79, no. 2, pp. 677-732, 2007.

[22] X. Zhou, J.-Y. Park, S. Huang, J. Liu, and P. L. McEuen, "Band structure, phonon scattering, and the performance limit of single-walled carbon nanotube transistors," Physical Review Letters, vol. 95, no. 14, Article ID 146805, 2005.

[23] T. Dürkop, S. A. Getty, E. Cobas, and M. S. Fuhrer, "Extraordinary mobility in semiconducting carbon nanotubes," Nano Letters, vol. 4, no. 1, pp. 35-39, 2004.

[24] J. Tersoff, "Contact resistance of carbon nanotubes," Applied Physics Letters, vol. 74, no. 15, pp. 2122-2124, 1999.

[25] M. J. O'Connell, Carbon Nanotubes: Properties and Applications, Taylor \& Francis, Oxford, UK, 2006.

[26] Z. Yao, C. L. Kane, and C. Dekker, "High-field electrical transport in single-wall carbon nanotubes," Physical Review Letters, vol. 84, no. 13, pp. 2941-2944, 2000.

[27] A. Javey, J. Guo, D. B. Farmer et al., "Self-aligned ballistic molecular transistors and electrically parallel nanotube arrays," Nano Letters, vol. 4, no. 7, pp. 1319-1322, 2004.

[28] A. Javey, J. Guo, M. Paulsson et al., "High-field quasiballistic transport in short carbon nanotubes," Physical Review Letters, vol. 92, no. 10, Article ID 106804, 2004.

[29] F. Léonard and J. Tersoff, "Role of fermi-level pinning in nanotube schottky diodes," Physical Review Letters, vol. 84, no. 20, pp. 4693-4696, 2000.
[30] Z. Chen, J. Appenzeller, J. Knoch, Y.-M. Lin, and P. Avouris, "The role of metal-nanotube contact in the performance of carbon nanotube field-effect transistors," Nano Letters, vol. 5, no. 7, pp. 1497-1502, 2005.

[31] P. Avouris, Z. Chen, and V. Perebeinos, "Carbon-based electronics," Nature Nanotechnology, vol. 2, no. 10, pp. 605-615, 2007.

[32] J. Guo, M. Lundstrom, and S. Datta, "Performance projections for ballistic carbon nanotube field-effect transistors," Applied Physics Letters, vol. 80, no. 17, pp. 3192-3194, 2002.

[33] M. Krüger, M. R. Buitelaar, T. Nussbaumer, C. Schönenberger, and L. Forró, "Electrochemical carbon nanotube field-effect transistor," Applied Physics Letters, vol. 78, no. 9, pp. 1291-1293, 2001.

[34] Z. Chen, D. Farmer, S. Xu, R. Gordon, P. Avouris, and J. Appenzeller, "Externally assembled gate-all-around carbon nanotube field-effect transistor," IEEE Electron Device Letters, vol. 29, no. 2, pp. 183-185, 2008.

[35] J. Appenzeller, J. Knoch, V. Derycke, R. Martel, S. Wind, and P. Avouris, "Field-modulated carrier transport in carbon nanotube transistors," Physical Review Letters, vol. 89, no. 12, Article ID 126801, 4 pages, 2002.

[36] M. Radosavljević, S. Heinze, J. Tersoff, and P. Avouris, "Drain voltage scaling in carbon nanotube transistors," Applied Physics Letters, vol. 83, no. 12, pp. 2435-2437, 2003.

[37] J. Appenzeller, M. Radosavljević, J. Knoch, and P. Avouris, "Tunneling versus thermionic emission in one-dimensional semiconductors," Physical Review Letters, vol. 92, no. 4, 4 pages, 2004.

[38] J. Nygård, D. H. Cobden, M. Bockrath, P. L. McEuen, and P. E. Lindelof, "Electrical transport measurements on single-walled carbon nanotubes," Applied Physics A, vol. 69, no. 3, pp. 297304, 1999.

[39] Y.-M. Lin, J. Appenzeller, and P. Avouris, "Ambipolar-tounipolar conversion of carbon nanotube transistors by gate structure engineering," Nano Letters, vol. 4, no. 5, pp. 947-950, 2004.

[40] S. Rosenblatt, H. Lin, V. Sazonova, S. Tiwari, and P. L. McEuen, "Mixing at $50 \mathrm{GHz}$ using a single-walled carbon nanotube transistor," Applied Physics Letters, vol. 87, no. 15, Article ID 153111, 3 pages, 2005.

[41] P. J. Burke, "AC performance of nanoelectronics: towards a ballistic THz nanotube transistor," Solid-State Electronics, vol. 48, no. 10-11, pp. 1981-1986, 2004.

[42] M. Dequesnes, Z. Tang, and N. R. Aluru, "Static and dynamic analysis of carbon nanotube-based switches," Journal of Engineering Materials and Technology, Transactions of the ASME, vol. 126, no. 3, pp. 230-237, 2004.

[43] D. A. Walters, L. M. Ericson, M. J. Casavant et al., "Elastic strain of freely suspended single-wall carbon nanotube ropes," Applied Physics Letters, vol. 74, no. 25, pp. 3803-3805, 1999.

[44] C. Stampfer, S. Rotter, and J. Burgdorfer, "Comment on dynamic range of nanotube- and nanowire-based electromechanical systems," Applied Physics Letters, vol. 88, no. 3, Article ID 036101, 2006.

[45] A. N. Cleland and M. L. Roukes, "Noise processes in nanomechanical resonators," Journal of Applied Physics, vol. 92, no. 5, pp. 2758-2769, 2002.

[46] H. W. C. Postma, I. Kozinsky, A. Husain, and M. L. Roukes, "Dynamic range of nanotube- and nanowire-based electromechanical systems," Applied Physics Letters, vol. 86, no. 22, Article ID 223105, 3 pages, 2005. 
[47] A. K. Hüttel, G. A. Steele, B. Witkamp, M. Poot, L. P. Kouwenhoven, and H. S. J. Van Der Zant, "Carbon nanotubes as ultrahigh quality factor mechanical resonators," Nano Letters, vol. 9, no. 7, pp. 2547-2552, 2009.

[48] H. M. Ouakad and M. I. Younis, "Nonlinear dynamics of electrically actuated carbon nanotube resonators," Journal of Computational and Nonlinear Dynamics, vol. 5, no. 1, Article ID 011009, 13 pages, 2010.

[49] C. Zhou, J. Kong, and H. Dai, "Intrinsic electrical properties of individual single-walled carbon nanotubes with small band gaps," Physical Review Letters, vol. 84, no. 24, pp. 5604-5607, 2000.

[50] L. Yang and J. Han, "Electronic structure of deformed carbon nanotubes," Physical Review Letter, vol. 85, no. 1, pp. 154-157, 2000.

[51] M. Huang, Y. Wu, B. Chandra et al., "Direct measurement of strain-induced changes in the band structure of carbon nanotubes," Physical Review Letters, vol. 100, no. 13, Article ID 136803, 2008.

[52] G. Y. Guo, L. Liu, K. C. Chu, C. S. Jayanthi, and S. Y. Wu, "Electromechanical responses of single-walled carbon nanotubes: Interplay between the strain-induced energy-gap opening and the pinning of the Fermi level," Journal of Applied Physics, vol. 98, no. 4, Article ID 044311, 4 pages, 2005.

[53] Y. Yoon and J. Guo, "Analysis of strain effects in ballistic carbon nanotube FETs," IEEE Transactions on Electron Devices, vol. 54, no. 6, pp. 1280-1287, 2007.

[54] C. Stampfer, A. Jungen, R. Linderman, D. Obergfell, S. Roth, and C. Hierold, "Nano-electromechanical displacement sensing based on single-walled carbon nanotubes," Nano Letters, vol. 6, no. 7, pp. 1449-1453, 2006.

[55] T. Helbling, Carbon Nanotube Field Effect Transistors As Electromechanical Transducers, vol. 8 of Scientific Reports on Micro and Nanosystems, Der Andere, Tönning, Germany, 2010.

[56] X. M. H. Huang, C. A. Zormant, M. Mehreganyt, and M. L. Roukes, "Nanoelectromehanical systems: nanodevice motion at microwave frequencies," Nature, vol. 421, no. 6922, article 496, 2003.

[57] D. W. Carr, L. Sekaric, and H. G. Craighead, "Measurement of nanomechanical resonant structures in single-crystal silicon," Journal of Vacuum Science and Technology B, vol. 16, no. 6, pp. 3821-3824, 1998.

[58] P. G. Collins, M. S. Fuhrer, and A. Zettl, "1/f noise in carbon nanotubes," Applied Physics Letters, vol. 76, no. 7, pp. 894-896, 2000.

[59] C. Kauth, M. Pastre, and M. Kayal, "Wideband low-noise rf front-end for cnt-nems sensors," in Proceedings of the Mixed Design of Integrated Circuits and Systems Conference, pp. 289293, IEEE, Warsaw, Poland, May 2012.

[60] C. Kauth, M. Pastre, and M. Kayal, "A self-regulating oscillator for sensor operation of nanoelectromechanical systems," in Proceedings of the New Circuits and Systems Conference, pp. 1-4, IEEE, Paris, France, June 2013. 

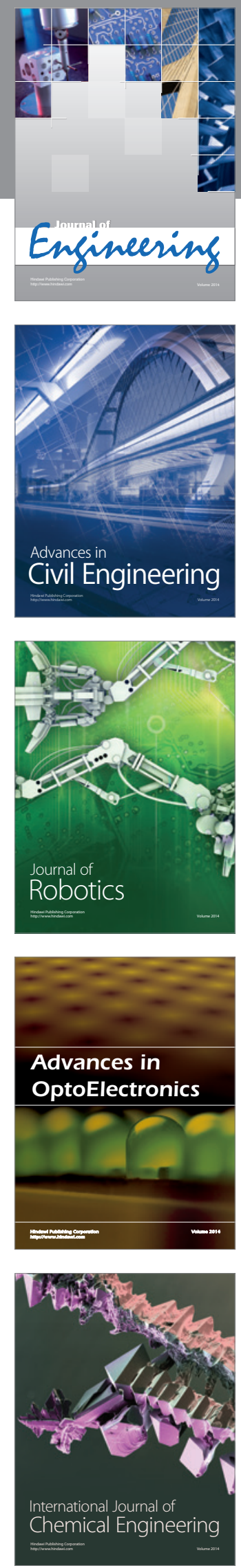

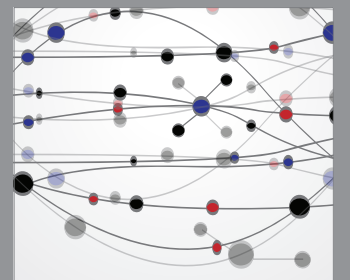

The Scientific World Journal
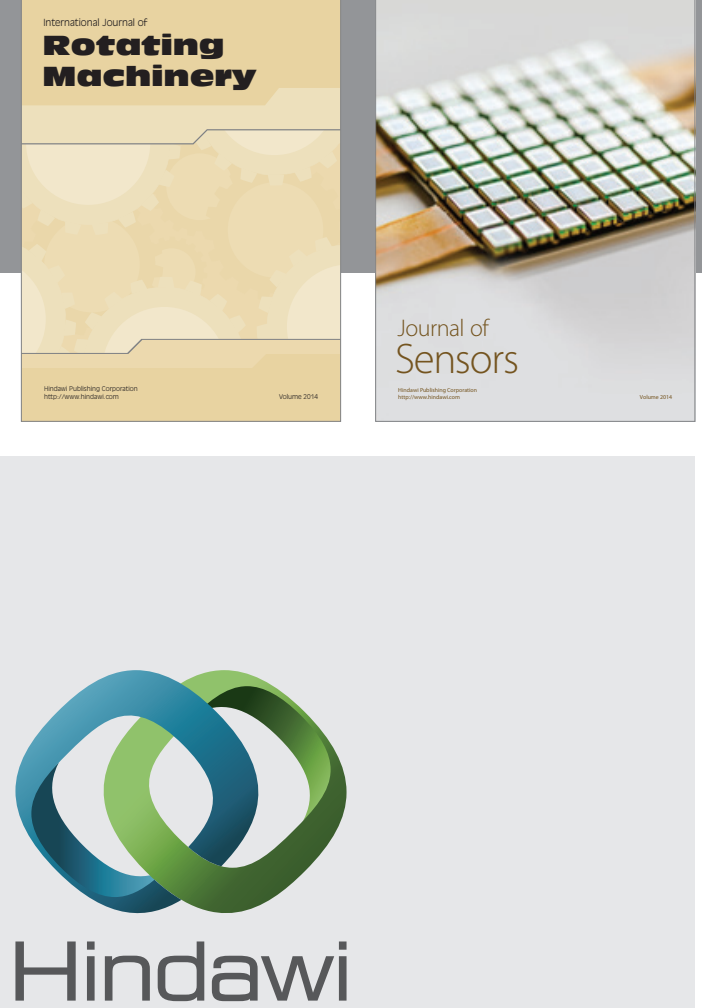

Submit your manuscripts at http://www.hindawi.com
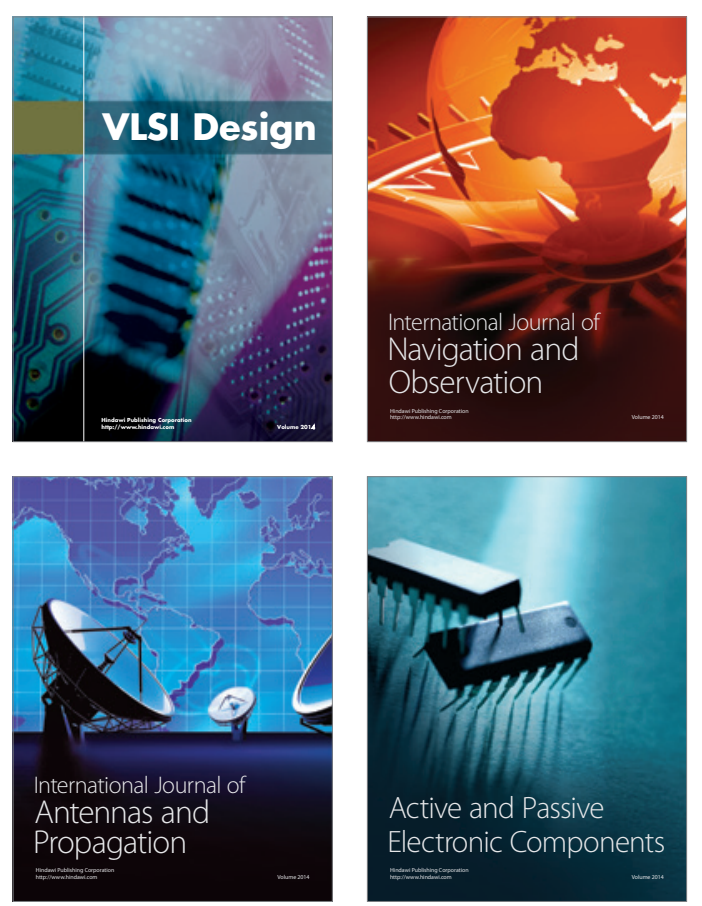
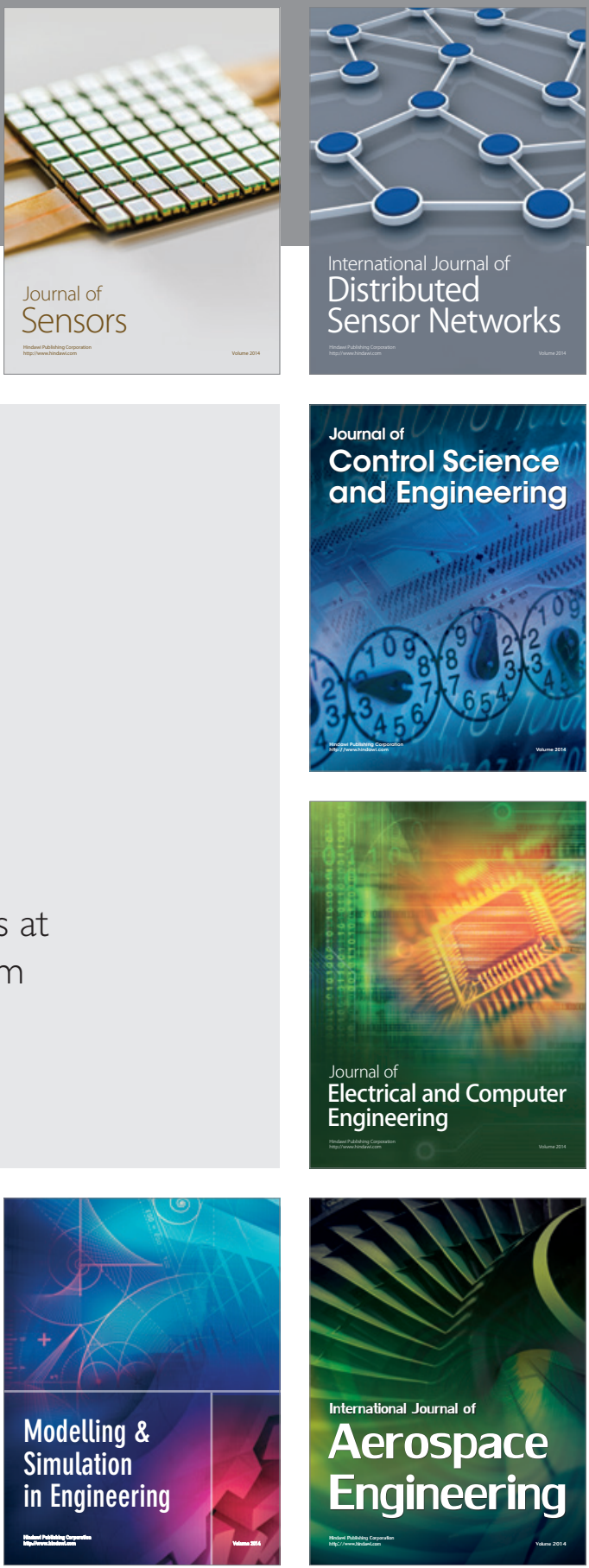

Journal of

Control Science

and Engineering
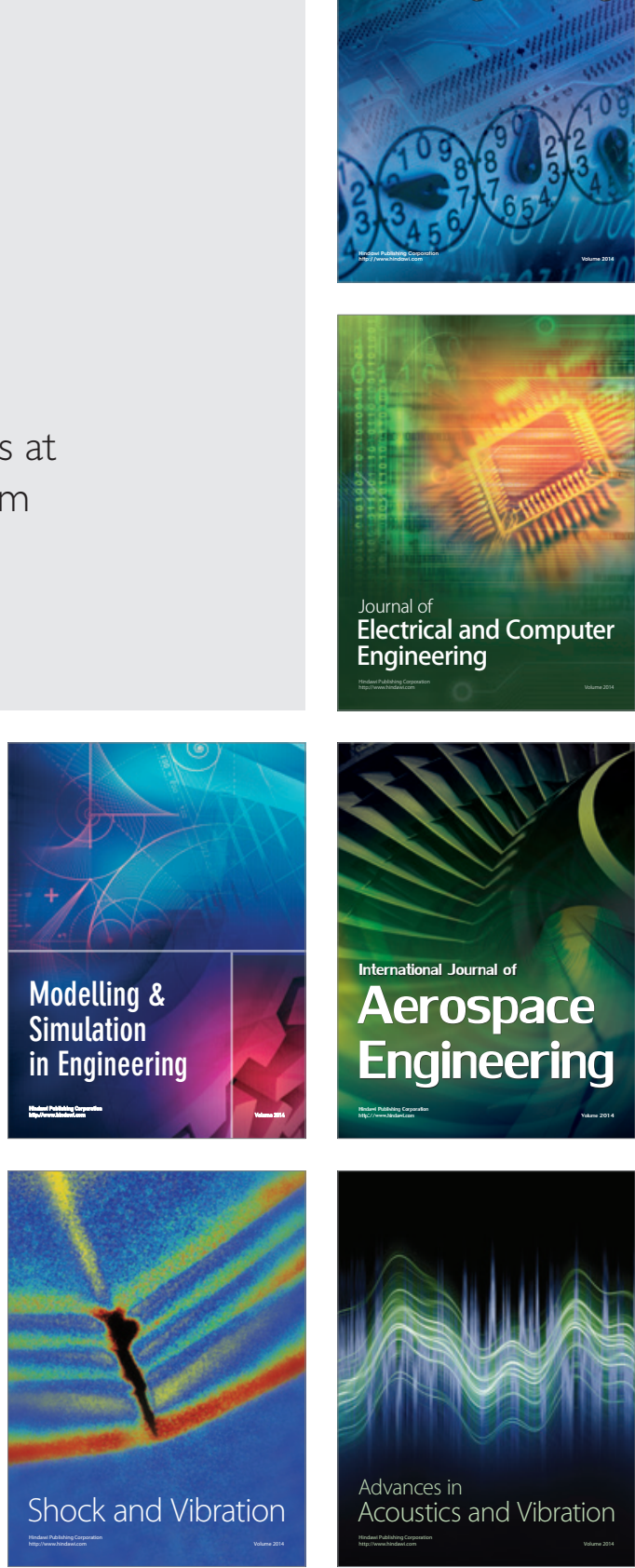